\title{
New Bounds and Tractable Instances for the Transposition Distance
}

\author{
Anthony Labarre
}

\begin{abstract}
The problem of sorting by transpositions asks for a sequence of adjacent interval exchanges that sorts a permutation and is of the shortest possible length. The distance of the permutation is defined as the length of such a sequence. Despite the apparently intuitive nature of this problem, introduced in 1995 by Bafna and Pevzner, the complexity of both finding an optimal sequence and computing the distance remains open today. In this paper, we establish connections between two different graph representations of permutations, which allows us to compute the distance of a few nontrivial classes of permutations in linear time and space, bypassing the use of any graph structure. By showing that every permutation can be obtained from one of these classes, we prove a new tight upper bound on the transposition distance. Finally, we give improved bounds on some other families of permutations and prove formulas for computing the exact distance of other classes of permutations, again in polynomial time.
\end{abstract}

Index Terms-Genome rearrangements, permutations, sorting by transpositions.

\section{INTRODUCTION}

$\mathrm{T}$ HE genome rearrangement problem [1], [2] can be formulated as that of finding a sequence of evolutionary events that transforms a given genome into another given one and is of the shortest possible length. The distance between the two genomes is the length of such a sequence.

The model we are interested in applies to the case where the order of genes is known and where all genomes share the same set and number of genes (without duplications), which allows us to represent them using permutations. Only one operation is taken into account here: biological transpositions, which consist of displacing a block of contiguous elements. It is easy to show that the induced distance is indeed a distance on the set of all permutations (i.e., it satisfies the three usual axioms) and that it is leftinvariant: The distance between any two permutations $\pi$ and $\sigma$ of the same set equals the distance between $\sigma^{-1} \circ \pi$ and the identity permutation $\iota=\left(\begin{array}{llll}1 & 2 & \cdots & n\end{array}\right)$. We can therefore restrict our attention to the problem of sorting permutations by transpositions.

This problem was first introduced in 1995 by Bafna and Pevzner [3], [4] and the complexity of both sorting permutations and computing their distance, as well as the maximal value the latter can reach, is still open today. Several authors have proposed polynomial-time approximation algorithms (whose best approximation ratio has long been $\frac{3}{2}$ [4], [5], [6], until Elias and Hartman [7] recently proposed a new $\frac{11}{8}$-approximation) as well as heuristics (see [5], [8], [9], [10]).

In this paper, we establish connections between the common graph of a permutation and the "cycle graph"

- The author is with the Département de Mathématique, Université Libre de Bruxelles, CP 216, Service de Géométrie, Combinatoire et Théorie des Groupes, Boulevard du Triomphe, B-1050 Bruxelles, Belgium.

E-mail: alabarre@ulb.ac.be.

Manuscript received 14 Feb. 2006; revised 25 Apr. 2006; accepted 28 Apr. 2006; published online 31 Oct. 2006.

For information on obtaining reprints of this article, please send e-mail to: tcbb@computer.org, and reference IEEECS Log Number TCBBSI-0014-0206. introduced in [4]. Use of the former was mentioned in [11] and led to a formula for computing another rearrangement distance in [12]. As we suspected, it proved fruitful for our problem too: The connections between the two graphs allowed us to compute the distance of a few nontrivial classes of permutations, bypass the use of any graph structure, prove a new tight upper bound on the transposition distance, and improve that upper bound in some other cases.

This paper is organized as follows: In Section 2, we review previous results and typical notations. In Section 3, we introduce a graph that we use in Section 4 to provide a formula for computing the distance of some special permutations. In Section 5, we use those permutations to derive an upper bound on the transposition distance of every permutation. Experimental data, comparisons, and heuristic improvements of this bound are discussed in Section 6 . We then turn to the study of other permutations in Sections 7 and 8, for which we can either compute the transposition distance or improve our upper bound on it. Finally, we discuss our results in Section 9 and suggest some open questions of interest.

A preliminary version of this work was presented at the Fifth Workshop on Algorithms in Bioinformatics (WABI '05) in Palma de Mallorca, Spain [13]. The main additions in this extended version consist of, besides changes in the structure and presentation, additional experimental data, three new sections (Sections 6, 7, and 8), and Appendices A and B.

\section{Notations and Preliminaries}

The symmetric group $S_{n}$ is the set of all permutations of $\{1,2, \ldots, n\}$; these are denoted by lowercase Greek letters, typically $\pi=\left(\pi_{1} \pi_{2} \cdots \pi_{n}\right)$, with $\pi_{i}=\pi(i)$.

\subsection{Transpositions and the Cycle Graph}

Definition 2.1. For any $\pi$ in $S_{n}$, the transposition $\tau(i, j, k)$ with $1 \leq i<j<k \leq n+1$ applied to $\pi$ exchanges the closed intervals determined, respectively, by $i$ and $j-1$ and by $j$ and 


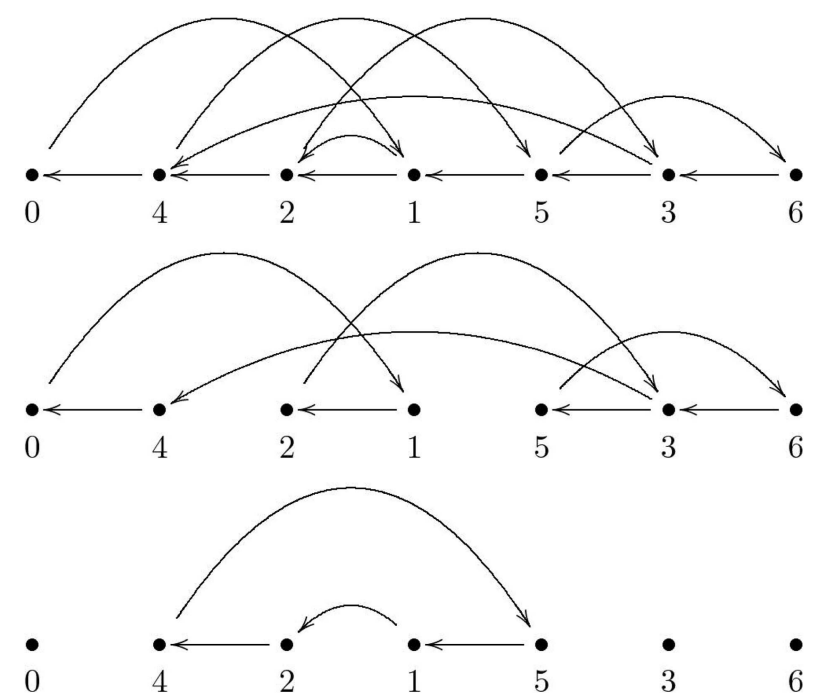

Fig. 1. The cycle graph of (4 2153 ) and its decomposition into two cycles.

$k-1$, transforming $\pi$ into $\pi \circ \tau(i, j, k)$. So, $\tau(i, j, k)$ is the following permutation:

$$
\left(\begin{array}{llll}
1 \cdots i-1 & i \cdots j-1 & j \cdots k-1 & k \cdots n \\
1 \cdots i-1 & j \cdots k-1 & i \cdots j-1 & k \cdots n
\end{array}\right) .
$$

Definition 2.2. The cycle graph of $\pi$ in $S_{n}$ is the bicolored directed graph $G(\pi)$ whose vertex set $\left(\pi_{0}=0, \pi_{1}, \ldots, \pi_{n}, \pi_{n+1}=\right.$ $n+1)$ is ordered by positions and whose edge set consists of:

- black edges $\left(\pi_{i}, \pi_{i-1}\right)$ for $1 \leq i \leq n+1$ and

- gray edges $\left(\pi_{i}, \pi_{i}+1\right)$ for $0 \leq i \leq n$.

The set of black and gray edges decomposes in a single way into alternating cycles, i.e., cycles which alternate black and gray edges, and we note the number of such cycles $c(G(\pi))$. Fig. 1 shows an example of a cycle graph, together with its decomposition.

Definition 2.3. The length of an alternating cycle in $G$ is the number of black edges it contains and a $k$-cycle in $G$ is an alternating cycle of length $k$.

Definition 2.4. A $k$-cycle in $G$ is odd (respectively, even) if $k$ is odd (respectively, even), and we note $c_{\text {odd }}(G(\pi))$ (respectively, $c_{\text {even }}(G(\pi))$ ) the number of odd (respectively, even) alternating cycles in $G(\pi)$.

Bafna and Pevzner [4] proved the following lower bound on the transposition distance, hereafter denoted by $d(\pi)$.

Theorem 2.1 [4]. For all $\pi$ in $S_{n}$ :

$$
d(\pi) \geq\left(n+1-c_{\text {odd }}(G(\pi))\right) / 2 .
$$

Definition 2.5. A cycle in $G$ is unoriented if it contains exactly one gray edge directed from left to right and oriented otherwise.
For instance, the first cycle in the decomposition of the graph of Fig. 1 is oriented; the second is not. A transposition $\tau(i, j, k)$ is said to act on black edges coming out of vertices $\pi_{i}, \pi_{j}$, and $\pi_{k}$ in $G(\pi)$. By extension, a transposition acts on one cycle (respectively, on two or three cycles) if all three black edges belong to that cycle (respectively, to those two or three cycles).

Definition 2.6. For a permutation $\pi$, a $k$-move is a transposition $\tau$ such that $c(G(\pi \circ \tau))=c(G(\pi))+k$.

Lemma 2.1 [4]. A transposition that acts on exactly two cycles in $G$ is a 0 -move.

Two alternating cycles can interact in several different ways, which we define below. To every alternating cycle $C$ in a cycle graph $G$, associate an interval $I_{C}$ defined by the minimum and maximum indices of the vertices that belong to $C$.

Definition 2.7. A cycle $C_{1}$ contains a cycle $C_{2}$ if $I_{C_{1}} \supset I_{C_{2}}$ and no black edge of $C_{1}$ belongs to $I_{C_{2}}$.

Definition 2.8. Two alternating cycles, $C_{1}, C_{2}$, cross if they do not contain each other and at least one black edge of $C_{1}$ (respectively, $C_{2}$ ) belongs to $I_{C_{2}}$ (respectively, $I_{C_{1}}$ ).

Definition 2.9. Two alternating cycles, $C_{1}, C_{2}$, interleave if, when reading the black edges of $C_{1}$ and $C_{2}$ from left to right, we alternately get a black edge from either cycle.

\subsection{Reduced Permutations}

Definition 2.10. For a permutation $\pi$, an ordered pair $\left(\pi_{i}, \pi_{i+1}\right)$ is a breakpoint if $\pi_{i+1} \neq \pi_{i}+1$ and an adjacency otherwise. The number of breakpoints of $\pi$ is denoted by $b(\pi)$.

Definition 2.11. A permutation $\pi$ in $S_{n}$ is reduced if $b(\pi)=n-1, \pi_{1} \neq 1$, and $\pi_{n} \neq n$.

Christie [5] shows that every permutation can be uniquely transformed into a reduced permutation without affecting its distance. The transformation of a permutation $\pi$ into its reduced version $g l(\pi)$ consists of decomposing $\pi$ into $r$ strips, which are maximal intervals containing no breakpoint, then removing strip 1 if it begins with 1 , strip $r$ if it ends with $n$, replacing every other strip with its minimal element, and, finally, renumbering the resulting sequence so as to obtain a new permutation of a possibly smaller set. Since an adjacency is a 1-cycle in $G$, a reduced permutation can also be defined as one whose cycle graph has no 1-cycles. ${ }^{1}$

Definition 2.12. Two permutations, $\pi$ and $\sigma$, are equivalent by reduction if $g l(\pi)=g l(\sigma)$, which we also write as $\pi \equiv_{r} \sigma$.

Theorem 2.2 [5]. For any two permutations $\pi$ and $\sigma$, if $\pi \equiv_{r} \sigma$, then $d(\pi)=d(\sigma)$.

\subsection{Toric Permutations}

Eriksson et al. [14] introduced an equivalence relation on $S_{n}$ whose equivalence classes are called toric permutations and which we define using Hultman's notations [15].

1. Note that $g l(\iota)$ is not defined because every element would have to be removed. 
Definition 2.13. The circular permutation obtained from a permutation $\pi$ in $S_{n}$ is $\pi^{\circ}=0 \pi_{1} \pi_{2} \cdots \pi_{n}$, with indices taken modulo $n+1$ so that $0=\pi_{0}^{\circ}=\pi_{n+1}^{\circ}$.

This circular permutation can be read starting from any position and the original linear permutation is reconstructed by taking the element following 0 as $\pi_{1}$ and removing 0 . For $x$ in $\{0,1,2, \ldots, n\}$, let $\bar{x}^{m}=(x+m)(\bmod n+1)$ and define the following operation on circular permutations:

$$
m+\pi^{\circ}=\overline{0}^{m}{\overline{\pi_{1}}}^{m}{\overline{\pi_{2}}}^{m} \ldots{\overline{\pi_{n}}}^{m} .
$$

Definition 2.14. For any $\pi$ in $S_{n}$, the toric permutation $\pi_{\circ}^{\circ}$ is the set of permutations in $S_{n}$ reconstructed from all circular permutations $m+\pi^{\circ}$ with $0 \leq m \leq n$.

Definition 2.15. Two permutations, $\pi, \sigma$, in $S_{n}$ are torically equivalent if $\sigma \in \pi_{\circ}^{\circ}$ (or $\pi \in \sigma_{\circ}^{\circ}$ ), which we also write as $\pi \equiv_{\circ}^{\circ} \sigma$.

The following property is the main reason why toric permutations were introduced:

Lemma 2.2 [14]. For all $\pi, \sigma$ in $S_{n}$ :

$$
\pi \equiv_{\circ}^{\circ} \sigma \Rightarrow d(\pi)=d(\sigma) \text {. }
$$

Another interesting, related result has been proved by Hultman [15].

Lemma 2.3 [15]. For all $\pi$ in $S_{n}$ and $0 \leq m \leq n$ : Every cycle in $G(\pi)$ is a cycle in $G(\sigma)$, where $\sigma$ is the permutation obtained from $\pi^{\circ}+m$.

\subsection{Known Upper Bounds}

We conclude this section with all upper bounds on the transposition distance we know of.

Theorem 2.3 [4]. For all $\pi$ in $S_{n}$ :

$$
d(\pi) \leq n+1-c(G(\pi)) .
$$

Theorem 2.4 [4]. For all $\pi$ in $S_{n}$ :

$$
d(\pi) \leq 3\left(n+1-c_{\text {odd }}(G(\pi))\right) / 4 .
$$

Theorem 2.5 [16]. For all $\pi$ in $S_{n}$ :

$$
d(\pi) \leq 3 b(\pi) / 4 .
$$

Theorem 2.6 [14]. For all $\pi$ in $S_{n}$ :

$$
d(\pi) \leq\left\{\begin{array}{cc}
\lceil 2 n / 3\rceil & \text { if } n<9 ; \\
\lfloor(2 n-2) / 3\rfloor & \text { if } n \geq 9 .
\end{array}\right.
$$

Elias and Hartman [7] proved upper bounds on the distance of three special classes of permutations. ${ }^{2}$

Definition 2.16. A permutation $\pi$ in $S_{n}$ is simple if $G(\pi)$ contains no cycle of length greater than three.

2. The definitions we give here are not the ones introduced by Hannenhalli and Pevzner [17] and Elias and Hartman [7], but we prove the equivalence between our definitions and theirs in Appendix B.

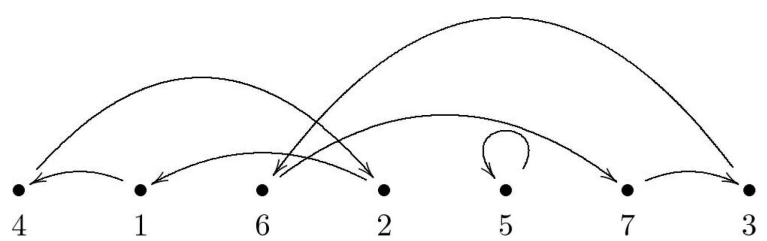

Fig. 2. The $\Gamma$-graph of the permutation (4 16257 3).

Definition 2.17. A permutation $\pi$ in $S_{n}$ is a 2-permutation (respectively, 3-permutation) if all cycles in $G(\pi)$ are of length 2 (respectively, 3).

Note that a 2-permutation (respectively, 3-permutation) only exists if $n+1$ can be divided by 4 (respectively, 3 ).

Theorem 2.7 [7]. For every simple permutation $\pi$ in $S_{n}$ which is neither a 2-permutation nor a 3-permutation:

$$
d(\pi) \leq\lfloor(n+1) / 2\rfloor .
$$

Theorem 2.8 [7]. For every 2-permutation $\pi$ in $S_{n}$ :

$$
d(\pi) \leq(n+1) / 2 .
$$

Theorem 2.9 [7]. For every 3-permutation $\pi$ in $S_{n}$ :

$$
d(\pi) \leq 11\left\lfloor\frac{n+1}{24}\right\rfloor+\left\lfloor\frac{3\left(\frac{n+1}{3} \bmod 8\right)}{2}\right\rfloor+1 .
$$

\section{Another Useful Graph}

We introduce a slight variant of the well-known graph of a permutation.

Definition 3.1. The $\Gamma$-graph of a permutation $\pi$ in $S_{n}$ is the directed graph $\Gamma(\pi)$ with ordered vertex set $\left(\pi_{1}, \ldots, \pi_{n}\right)$ and edge set $\left\{\left(\pi_{i}, \pi_{j}\right) \mid \pi_{i}=j\right\}$.

Fig. 2 shows an example of a $\Gamma$-graph. If $C=\left(i_{1}, i_{2}, \ldots, i_{k}\right)$ is a cycle of $\pi$ (i.e., $\pi$ maps $i_{l}$ onto $i_{l+1}$ for $1 \leq l \leq k-1$ and $i_{k}$ onto $\left.i_{1}\right)$, we obtain a cycle $\left(\pi_{i_{1}}, \pi_{i_{2}}, \ldots, \pi_{i_{k}}\right)$, which we also denote $C$, in $\Gamma(\pi)$, and call it a $k$-cycle. The length of a cycle in $\Gamma$ is therefore $k$.

Definition 3.2. A $k$-cycle in $\Gamma$ is increasing (respectively, decreasing) if $k \geq 3$ and its elements can be written as an increasing (respectively, decreasing) sequence and nonmonotonic otherwise.

A cycle that is either increasing or decreasing is also referred to as monotonic. For instance, in Fig. 2, cycle $(4,2,1)$ is decreasing, cycle (5) is nonmonotonic, and cycle $(3,6,7)$ is increasing. In a quite similar fashion to the parity of cycles defined in the context of $G$, a $k$-cycle in $\Gamma$ is odd (respectively, even) if $k$ is odd (respectively, even). Likewise, $c(\Gamma(\pi))$ denotes the number of cycles in $\Gamma(\pi)$, and $c_{\text {odd }}(\Gamma(\pi))$ (respectively, $c_{\text {even }}(\Gamma(\pi))$ ) denotes the number of odd (respectively, even) cycles in $\Gamma(\pi)$. Finally, note that Definitions $2.7,2.8$, and 2.9 naturally adapt to the $\Gamma$-graph. 


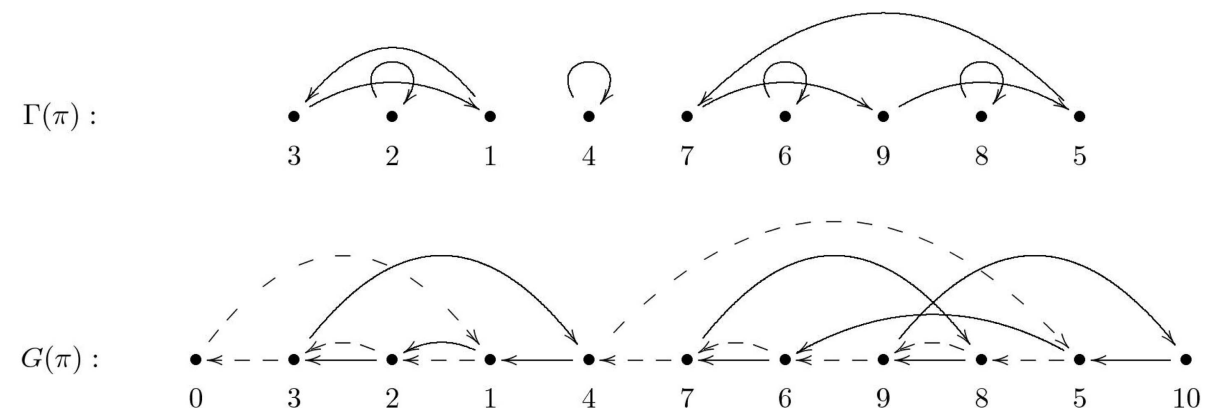

Fig. 3. Illustration of Proposition 4.1 and Observation 4.1.

\section{An EXPLICIT FORMULA FOR SOME Permutations}

Definition 4.1. $A$-permutation is a reduced permutation that fixes even elements (thus $n$ must be odd).

An example of a $\gamma$-permutation is (3 21476985 ). We will show (Proposition 4.5) that the distance of such a permutation can be computed quickly, without the need for any graph structure.

Proposition 4.1. For every $\gamma$-permutation $\pi$ in $S_{n}$ :

$$
\left\{\begin{array}{l}
c_{\text {even }}(G(\pi))=2 c_{\text {even }}(\Gamma(\pi)) \\
c_{\text {odd }}(G(\pi))=2\left(c_{\text {odd }}(\Gamma(\pi))-\frac{n-1}{2}\right) .
\end{array}\right.
$$

Proof. Each vertex $\pi_{i}$ of $\Gamma(\pi)$, with $i$ odd, is both the starting point of an edge $\left(\pi_{i}, \pi_{j_{1}}\right)$ and the ending point of an edge $\left(\pi_{j_{2}}, \pi_{i}\right)$. From our definitions, $\pi_{i}+1$ is mapped onto itself since it is even and $\pi_{j_{1}}$ precedes $\pi_{i}+1$ in $\Gamma(\pi)$. In $G(\pi)$, those edges are each transformed, as explained below, into one sequence of two edges (gray-black for the first one, black-gray for the second one):

- $\left(\pi_{i}, \pi_{j_{1}}\right)$ becomes $\left(\pi_{i}, \pi_{i}+1\right),\left(\pi_{i}+1, \pi_{j_{1}}\right)$ and

- $\left(\pi_{j_{2}}, \pi_{i}\right)$ becomes $\left(\pi_{i}, \pi_{i-1}\right),\left(\pi_{i-1}, \pi_{j_{2}}\right)$,

i.e., $\left(\pi_{i}, \pi_{j_{1}}\right)$ is transformed in one of the following ways (depending on the relative positions of $\pi_{i}$ and $\pi_{j_{1}}$ ):

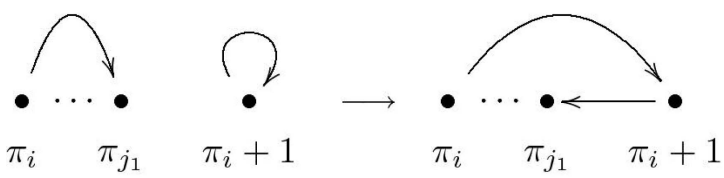

(a)

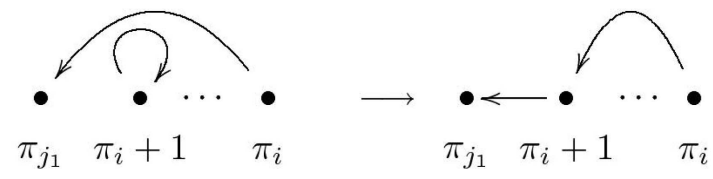

(b)

By definition of $\Gamma$, we know that $\pi_{j_{2}}=i$. Since $\pi_{i-1}=i-1$, the edge $\left(\pi_{j_{2}}, \pi_{i}\right)$ is transformed in one of the following ways (depending on the relative positions of $\pi_{i}$ and $\pi_{j_{2}}$ ):

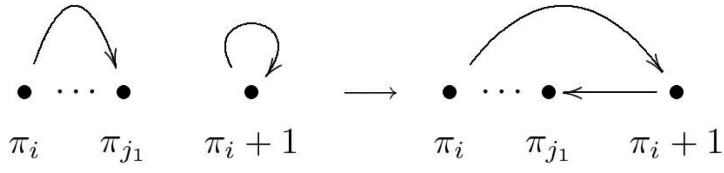

(a)
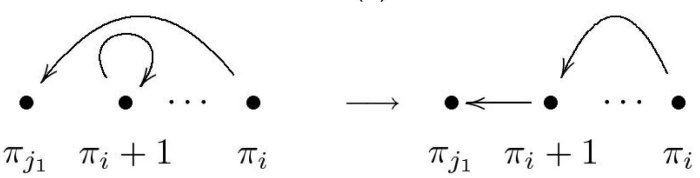

(b)

Therefore, each $k$-cycle $(k \geq 2)$ in $\Gamma(\pi)$ provides two alternating $k$-cycles in $G(\pi)$, one of which actually corresponds to the backward course of the cycle in $\Gamma(\pi)$. Finally, 1-cycles in $\Gamma(\pi)$ are not preserved in $G(\pi)$ and there are $\frac{n-1}{2}$ of them.

The next observation follows naturally from our transformation.

Observation 4.1. For a $\gamma$-permutation $\pi$, the two alternating cycles $C_{1}, C_{2}$ in $G(\pi)$ that correspond to a $k$-cycle $C$ in $\Gamma(\pi)$ interleave. Moreover:

1. If $k=2$, then $C_{1}$ and $C_{2}$ are unoriented.

2. If $C$ is monotonic, then either $C_{1}$ or $C_{2}$ is oriented.

3. If $C$ is nonmonotonic and $k \geq 4$, then both $C_{1}$ and $C_{2}$ are oriented.

Fig. 3 illustrates Proposition 4.1 and Observation 4.1. We derive the following lower bound from Proposition 4.1 and Theorem 2.1:

Lemma 4.1. For every $\gamma$-permutation $\pi$ in $S_{n}$, we have $d(\pi) \geq n-c_{\text {odd }}(\Gamma(\pi))$.

Proof. Straightforward.

We first study $\gamma$-permutations such that $\Gamma$ has only one "long" $k$-cycle (i.e., with $k>1$ ), distinguishing between monotonic cycles and nonmonotonic ones.

\subsection{Monotonic Cycles}

Definition 4.2. An $\alpha$-permutation is a reduced permutation that fixes even elements and whose $\frac{n+1}{2}$ odd elements form one monotonic cycle in $\Gamma$, referred to as its main cycle. 
An example of an $\alpha$-permutation for $n=7$ is (3254761). Note that, for fixed $n$, there are only two $\alpha$-permutations in $S_{n}$ : One has an increasing main cycle, and the other has a decreasing main cycle. Therefore, the only other $\alpha$-permutation, for $n=7$, is (7 211436 5), which is the inverse of the above example.

Proposition 4.2. For every $\alpha$-permutation $\pi$ in $S_{n}$, we have

$$
d(\pi)=n-c_{\text {odd }}(\Gamma(\pi))=|C|-(|C| \bmod 2),
$$

where $|C|=\frac{n+1}{2}$ is the number of elements in its main cycle $C$.

Proof. Every $\alpha$-permutation is a $\gamma$-permutation, so $d(\pi) \geq$ $|C|-(|C| \bmod 2)$ (Lemma 4.1). Assume that $C$ is increasing (a similar proof is easily obtained in the decreasing case) and consider transpositions $\tau_{1}(2,4, n+1), \tau_{2}(1,3, n)$, $\tau_{3}(2,3, n+1)$, and $\tau_{4}(1,2, n+1)$. If $|C|$ is odd, then an optimal sorting sequence of length $|C|-1$ for $\pi$ is obtained by applying $\tau_{2} \circ \tau_{1}$ exactly $\frac{|C|-1}{2}$ times. If $|C|$ is even, then an optimal sorting sequence of length $|C|$ for $\pi$ is obtained by applying $\tau_{2} \circ \tau_{1}$ exactly $\frac{|C|-2}{2}$ times, then $\tau_{3}$ and, finally, $\tau_{4}$. The proof that those sequences indeed sort $\pi$ is given in Appendix A.

\subsection{Nonmonotonic Cycles}

Definition 4.3. A $\beta$-permutation is a reduced permutation that fixes even elements and whose odd elements form one nonmonotonic cycle in $\Gamma$.

We now show that Proposition 4.2 still holds if the main cycle of $\Gamma$ is nonmonotonic. We use so-called exchanges in order to simplify the proofs, thus bypassing the construction of optimal sequences of transpositions.

Definition 4.4. An exchange exc $(i, j)$ is the permutation that exchanges elements in positions $i$ and $j$, thus transforming every permutation $\pi$ into the permutation $\pi \circ \operatorname{exc}(i, j)$. So, $\operatorname{exc}(i, j)$ is the following permutation:

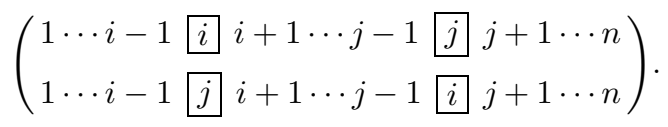

We only use exchanges of the form $\operatorname{exc}(i, i+2 k)$ with $k \geq 1$; such an exchange has the same effect as two transpositions, but the correspondence between those two types of operations is not that straightforward when exchanges are composed.

Definition 4.5. Two edges in $\Gamma(\pi)$ cross if the intervals determined by their endpoints do not contain each other and have a nonempty intersection.

Fig. 4 shows the four possible configurations for two crossing edges. Clearly, for every $\beta$-permutation $\pi$ (except (3 2 1)), the main cycle of $\Gamma(\pi)$ contains crossing edges. We are going to transform $\pi$ into a permutation $\sigma$ that reduces to an $\alpha$-permutation by removing crossing edges using a certain sequence $\mathcal{E}$ of exchanges. This yields the following upper bound on the distance of a $\beta$-permutation $\pi$ :

$$
d(\pi) \leq f(\mathcal{E})+d(\sigma),
$$

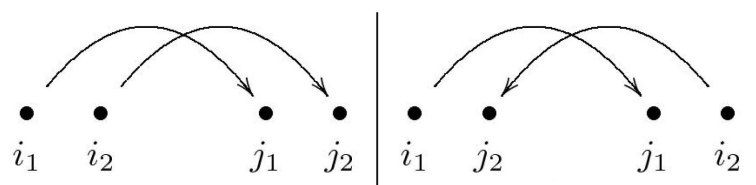

(a)

(b)

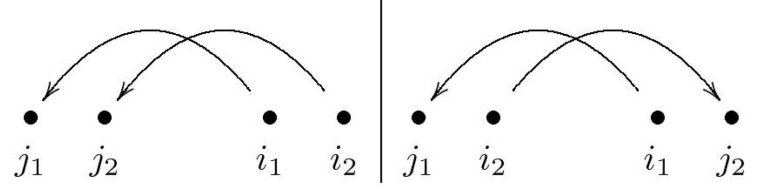

(c)

(d)

Fig. 4. The four possible configurations for crossing edges in $\Gamma(\pi)$.

where $f(\mathcal{E})$ gives the minimum number of transpositions having the same effect on $\pi$ as $\mathcal{E}$ does. Finding some $\sigma$ is not difficult, but we have to find a $\sigma$ such that our upper bound in (8) is minimized.

Eliminating a crossing can be done by making the ending point of one edge become the starting point of the one it crosses, and this will be achieved using a sequence of exchanges of the form described in the following proposition:

Proposition 4.3. For both sequences $\mathcal{E}=\operatorname{exc}(i, i+2) \circ$ $\operatorname{exc}(i, i+4) \circ \cdots \circ \operatorname{exc}(i, i+2 t)$ and $\mathcal{F}=\operatorname{exc}(i, i+2 t) \circ$ ․ $\operatorname{exc}(i, i+4) \circ \operatorname{exc}(i, i+2)$ of $t$ exchanges:

$$
f(\mathcal{E})=f(\mathcal{F})=t+(t \bmod 2) .
$$

Proof. Both sequences, when applied to the identity permutation, result in a permutation $\pi$ which contains one long cycle and whose other cycles are all fixed points since they are never affected by any exchange. If $t=1$, then the long cycle is nonmonotonic, and it is easily seen that $d(\pi)=2$; otherwise, the long cycle of $\pi$ is increasing in the case of $\mathcal{E}$ and decreasing in the case of $\mathcal{F}$. All elements before position $i$ and after position $i+2 t$ are fixed and removing them transforms $\pi$ into $g l(\pi)$, which is an $\alpha$-permutation whose main cycle has $t+1$ elements. Therefore, by Theorem 2.2 and Proposition 4.2, we have:

$$
\begin{aligned}
d(\pi) & =t+1-((t+1) \bmod 2) \\
& =t+(t \bmod 2)=f(\mathcal{E})=f(\mathcal{F}) .
\end{aligned}
$$

By a path, we mean a sequence of edges joining the ending point $i$ of an edge to the starting point $j$ of the edge it crosses and such that the extremities of each edge in this path belong to the interval determined by $i$ and $j$. Furthermore, we will refer to the elimination of this path as its contraction. Let us now compute the distance of $\beta$-permutations.

Proposition 4.4. For every $\beta$-permutation $\pi$ in $S_{n}$, we have

$$
d(\pi)=n-c_{\text {odd }}(\Gamma(\pi))=|C|-(|C| \bmod 2),
$$

where $|C|=\frac{n+1}{2}$ is the number of elements in its main cycle $C$.

Proof. Every $\beta$-permutation is a $\gamma$-permutation, so $d(\pi) \geq$ $|C|-(|C| \bmod 2)$ (Lemma 4.1). If $\pi=\left(\begin{array}{lll}3 & 2 & 1\end{array}\right)$, we are done; otherwise, $C$ contains at least one crossing. 

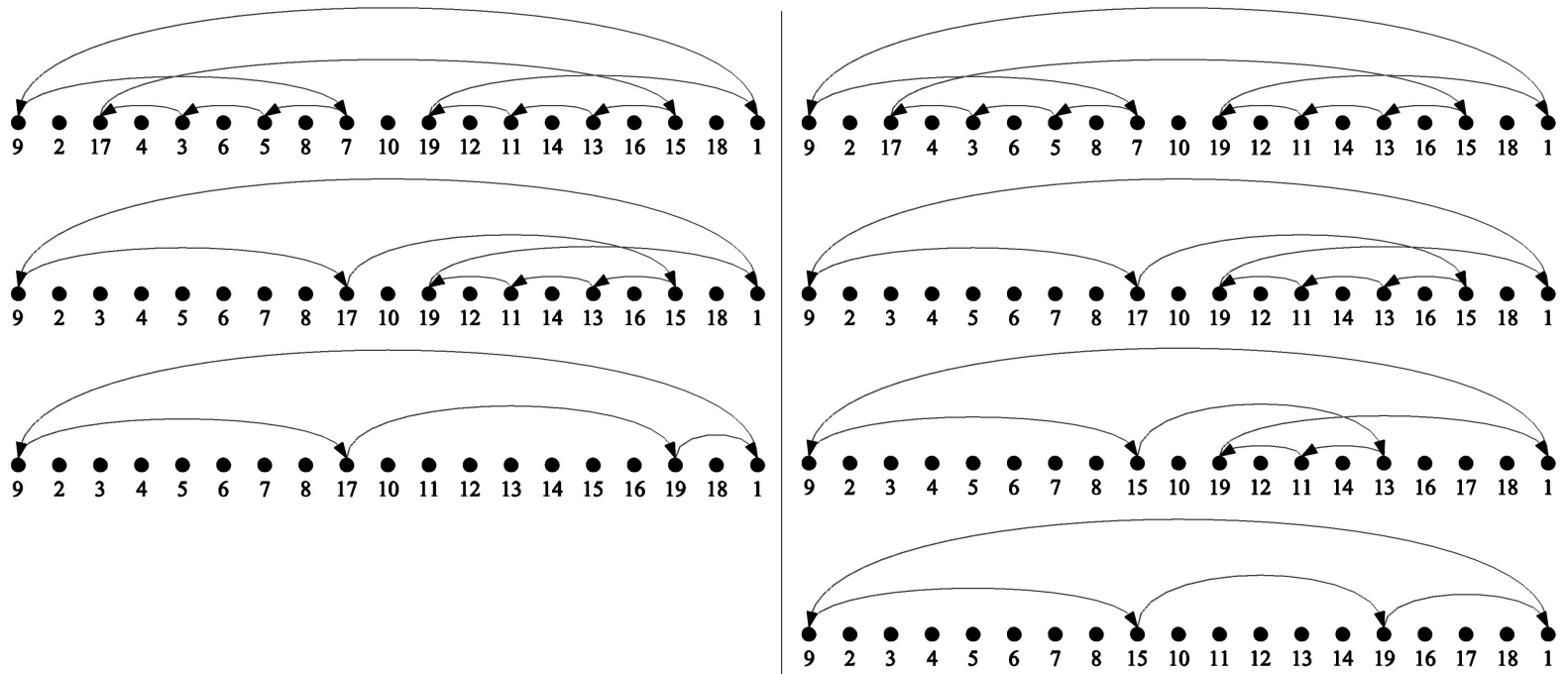

(a)

(b)

Fig. 5. Two ways of contracting paths in a $\beta$-permutation (1-cycles omitted for clarity).

In that case, there is a path of $t$ edges joining the two crossing edges; this path can be contracted by a sequence of $t$ exchanges, sorting the elements belonging to that part of the cycle. For instance, in Fig. 4a, it suffices to apply the sequence $\operatorname{exc}\left(i_{2}, j_{1}\right) \circ \cdots \circ \operatorname{exc}\left(i_{2}, i_{2}+4\right) \circ \operatorname{exc}\left(i_{2}, i_{2}+2\right)$ and those $t$ exchanges correspond to exactly $t+(t \bmod$ 2) transpositions (Proposition 4.3).

Once this path has been contracted, $t$ vertices have been removed from $C$ and this results in a permutation $\sigma$ reducible to an $\alpha$-permutation. Therefore,

$$
\begin{aligned}
d(\pi) & \leq d(\pi, \sigma)+d(\sigma) \\
& =t+(t \bmod 2)+|C|-t-((|C|-t) \bmod 2) \\
& =|C|-(|C| \bmod 2) .
\end{aligned}
$$

If there are $p$ paths of $t_{g}$ edges each $(1 \leq g \leq p)$, contracting them all "individually" takes $\sum_{g=1}^{p} t_{g}$ exchanges or $\sum_{g=1}^{p}\left(t_{g}+\left(t_{g} \bmod 2\right)\right)$ transpositions (Proposition 4.3). This can actually be improved by exchanging the last exchanged element in the first contracted path with the first element of the next path to contract, then continuing the contraction of the latter with dependent exchanges as before, repeating the same process whenever needed. For instance, Fig. 5 shows two different transformations of a $\beta$-permutation into a permutation reducible to an $\alpha$-permutation: Fig. 5a, which removes both crossings using two disjoint sequences, uses $3+3$ exchanges $=8$ transpositions (Proposition 4.3), whereas Fig. 5b, which removes both crossings using a single sequence, uses the same number of exchanges, but requiring only six transpositions this time.

Every $\beta$-permutation $\pi$ whose $\Gamma$-graph contains $p$ paths of $t_{g}$ edges to contract $(1 \leq g \leq p)$ can therefore be transformed into a permutation $\sigma$ reducible to an $\alpha$-permutation and such that $d(\pi, \sigma)=T+(T \bmod 2)$, where $T=\sum_{g=1}^{p} t_{g}$. The transformation removes $T$ vertices from $C$, which yields the following upper bound:

$$
\begin{aligned}
d(\pi) & \leq d(\pi, \sigma)+d(\sigma) \\
& =T+(T \bmod 2)+|C|-T-((|C|-T) \bmod 2) \\
& =|C|-(|C| \bmod 2)
\end{aligned}
$$

which equals the lower bound given above.

\subsection{Distance of $\gamma$-Permutations}

Each cycle in $\Gamma(\pi)$ can be sorted (by transpositions) individually so that the resulting permutation has the same $\Gamma$-graph as $\pi$, except that one cycle has been transformed into fixed points. This strategy yields the following upper bound on $d(\pi)$ :

Lemma 4.2. For every permutation $\pi$, consider its disjoint cycle decomposition $\Gamma(\pi)=C_{1} \cup C_{2} \cup \cdots \cup C_{c(\Gamma(\pi))}$. Denote $d(C)$ the minimum number of transpositions required to transform $C=\left(i_{1}, i_{2}, \ldots, i_{k}\right)$ into $\left(i_{1}\right),\left(i_{2}\right), \ldots,\left(i_{k}\right)$; then,

$$
d(\pi) \leq \sum_{i=1}^{c(\Gamma(\pi))} d\left(C_{i}\right)
$$

We now show that (9) is tight for $\gamma$-permutations.

Proposition 4.5. For every $\gamma$-permutation $\pi$ in $S_{n}$ :

$$
d(\pi)=n-c_{\text {odd }}(\Gamma(\pi))
$$

Proof. Denote $\operatorname{odd}(\Gamma(\pi))$ (respectively, even $(\Gamma(\pi))$ ) the set of odd (respectively, even) cycles in $\Gamma(\pi)$; Lemma 4.2 and Propositions 4.2 and 4.4 yield 


$$
\begin{aligned}
d(\pi) & \leq \sum_{i=1}^{c(\Gamma(\pi))}\left|C_{i}\right|-\left(\left|C_{i}\right| \bmod 2\right) \\
& =\sum_{C_{i_{1}} \in \text { odd }(\Gamma(\pi))}\left(\left|C_{i_{1}}\right|-1\right)+\sum_{C_{i_{2}} \in \operatorname{even}(\Gamma(\pi))}\left|C_{i_{2}}\right| \\
& =\sum_{i=1}^{c(\Gamma(\pi))}\left|C_{i}\right|-c_{\text {odd }}(\Gamma(\pi)) .
\end{aligned}
$$

Since every element belongs to exactly one cycle, the last sum equals $n$ and the proof follows from Lemma 4.1 .

This proposition actually leads to a more general result.

Theorem 4.1. Every permutation $\pi$ in $S_{n}$ that reduces to a $\gamma$-permutation has distance

$$
d(\pi)=n-c_{o d d}(\Gamma(\pi)) .
$$

Moreover, every permutation $\sigma$ with $n$ odd and whose odd elements occupy odd positions and form an increasing subsequence modulo $n+1$ can be transformed in linear time into a permutation $\pi$ such that $d(\sigma)=d(\pi)=n-c_{\text {odd }}(\Gamma(\pi))$.

Proof. Let $\pi$ be a $\gamma$-permutation in $S_{n}$ : transforming $\pi$ into a permutation $\sigma \neq \pi$ such that $\sigma \equiv_{r} \pi$ is done by creating adjacencies in $\pi$, i.e., repeatedly adding an element $e$ between $\pi_{i}$ and $\pi_{i+1}$ such that $e=\pi_{i}+1$ or $e=\pi_{i+1}-1$ (a subsequent renumbering of elements is, of course, required). Since either $\pi_{i}$ or $\pi_{i}+1$ is fixed (or possibly both, if this is not the first addition), adding $e$ comes down to inserting a new 1-cycle in $\Gamma(\pi)$, and this increases both $n$ and $c_{o d d}(\Gamma(\pi))$ by 1 at each step, so (10) still holds (Theorem 2.2).

For the second category, note that $\pi^{\circ} \pm 1$ fixes all even elements and therefore falls into the category discussed above. The proof follows from Lemma 2.2.

\section{A New Upper Bound}

We now show that the right-hand side of (10) is an upper bound on the transposition distance. First, we show why $\gamma$-permutations are so important.

Theorem 5.1. Every permutation $\pi$ in $S_{n}$, except $\iota$, can be obtained from a permutation $\sigma$ in $S_{n+k}$ that reduces to a $\gamma$-permutation.

Proof. If $\pi \neq \iota$ does not reduce to a $\gamma$-permutation, add a 1-cycle to $\Gamma(\pi)$ between every ordered pair $\left(\pi_{i}, \pi_{i+1}\right)(1 \leq i \leq n-1)$; then the resulting permutation $\sigma$ in $S_{n+k}$ reduces to a $\gamma$-permutation. The transformation can clearly be reverted, and this completes the proof.

Theorem 5.2. For all $\pi$ in $S_{n}$ :

$$
d(\pi) \leq n-c_{o d d}(\Gamma(\pi))
$$

Proof. If $\pi=\iota$, then the proof follows at once. Otherwise, let $\sigma$ be the permutation from which $\pi$ is obtained by removing $k$ 1-cycles from $\Gamma(\sigma)$, as described in Theorem 5.1. The sorting strategy of Lemma 4.2, optimal for $\sigma$, still works for $\pi$, only it may not be optimal anymore. Moreover, Theorem 4.1 gives the distance of $\sigma$. Therefore,

$$
\begin{aligned}
d(\pi) \leq d(\sigma) & =n+k-c_{\text {odd }}(\Gamma(\sigma)) \\
& =n+k-c_{\text {odd }}(\Gamma(\pi))-k \\
& =n-c_{\text {odd }}(\Gamma(\pi)) .
\end{aligned}
$$

\section{Tests and HeURistic Improvements OF the NeW UPPER BOUND}

Table 1 shows the number of cases where (11) is at least as good as the bounds given in Section 2. A first heuristic improvement can be obtained through torism.

Theorem 6.1. For all $\pi$ in $S_{n}$ :

$$
d(\pi) \leq n-\max _{\sigma \in \pi_{\circ}^{\circ}} c_{o d d}(\Gamma(\sigma)) .
$$

Proof. Straightforward from Theorem 5.2 and Lemma 2.2. $\square$

Experiments show (Table 1) that (12) is a substantial improvement over (11), but it is hard to express or evaluate this improvement because the evolution of $\Gamma$ under the toric equivalence relation does not seem easy to predict, whereas that of $G$ is well known (Lemma 2.3). Note, by the way, that the other upper bounds cannot be lowered through torism since neither the cycle graph structure nor the number of breakpoints will be affected.

A second heuristic improvement of (11) can be obtained through reduction.

Theorem 6.2. For all $\pi \neq \iota$ in $S_{n}$, let $g l(\pi)$ denote its reduced version in $S_{m}$, where $m \leq n$, then

$$
d(\pi) \leq m-\max _{\sigma \in(g l(\pi))_{\circ}^{\circ}} c_{o d d}(\Gamma(\sigma)) .
$$

All other bounds can take advantage of this reduction as well, except for (1), (2), and (3). This time, we do not compare (13) with other bounds; instead, for $1 \leq i \leq 9$, we generate all permutations with their distance and check how (13) overestimates their distance. Table 2 shows the results; for our range of experiments, it seems that (13) is a $\frac{3}{2}$-approximation.

\section{Perforations of $\alpha$-Permutations}

After looking at $\gamma$-permutations, it is natural to wonder how deleting their fixed points affects their distance. A careful analysis allows us to further improve (11) in the case of $\alpha$-permutations.

Note that deleting a 1-cycle in position $i$ in $\Gamma$ can be done by placing $\pi_{i}$ just before $\pi_{i}+1$ using a transposition, then removing the obtained adjacency and renumbering the other elements appropriately.

Definition 7.1. $A k$-perforation $\pi$ in $S_{n}$ of an $\alpha$-permutation $\sigma$ in $S_{n+k}$ is a permutation obtained by removing $k \geq 1$ 1-cycles from $\Gamma(\sigma)$ and renumbering the remaining elements.

For instance, a 3-perforation of the $\alpha$-permutation (325476 $9811101)$ is $(3[2547[6] 961101)=(24356871)$. Let us 
TABLE 1

Comparison of the New Upper Bounds with Previous Results

\begin{tabular}{r|r|r|r|r|r|r|r|r|r}
$n$ & $n !$ & $|(11) \leq(1)|$ & $|(12) \leq(1)|$ & $|(11) \leq(2)|$ & $|(12) \leq(2)|$ & $|(11) \leq(3)|$ & $|(12) \leq(3)|$ & $|(11) \leq(4)|$ & $|(12) \leq(4)|$ \\
\hline 1 & 1 & 1 & 1 & 1 & 1 & 1 & 1 & 1 & 1 \\
2 & 2 & 2 & 2 & 1 & 1 & 1 & 1 & 1 & 1 \\
3 & 6 & 6 & 6 & 2 & 2 & 1 & 1 & 6 & 6 \\
4 & 24 & 19 & 24 & 8 & 11 & 8 & 9 & 15 & 21 \\
5 & 120 & 101 & 112 & 45 & 60 & 24 & 36 & 31 & 54 \\
6 & 720 & 529 & 671 & 304 & 451 & 49 & 73 & 495 & 703 \\
7 & 5040 & 3837 & 4654 & 2055 & 3318 & 722 & 1336 & 1611 & 3574 \\
8 & 40320 & 28354 & 37209 & 17879 & 27486 & 3094 & 5957 & 4355 & 9864 \\
9 & 362880 & 257844 & 336744 & 104392 & 259195 & 60871 & 132801 & 10243 & 21610 \\
10 & 3628800 & 2469217 & 3280815 & 430164 & 1244002 & 361659 & 931584 & 485154 & 1376134 \\
\hline \hline$n$ & $\mid$ simple permutations & $\mid 2-p e r m u t a t i o n s$ & $\mid 3-p e r m u t a t i o n s$ & $|(11) \leq(5)|$ & $|(12) \leq(5)|$ & $|(11) \leq(6)|$ & $|(12) \leq(6)|$ & $|(11) \leq(7)|$ & $|(12) \leq(7)|$ \\
\hline 1 & 1 & - & - & 1 & 1 & - & - & - & - \\
2 & 2 & - & 1 & 1 & 1 & - & - & 1 & 1 \\
3 & 6 & 1 & - & 6 & 6 & 1 & 1 & - & - \\
4 & 16 & - & - & 11 & 16 & - & - & - & - \\
5 & 48 & - & 12 & 19 & 30 & - & - & 12 & 12 \\
6 & 204 & - & - & 32 & 50 & - & - & - & - \\
7 & 876 & 21 & - & 369 & 192 & 9 & 21 & - & - \\
8 & 3636 & - & 464 & 749 & 1530 & - & - & 10 & 20 \\
9 & 18756 & - & - & 1433 & 2781 & - & - & - & - \\
10 & 105480 & - & 2678 & 4896 & - & - & - & - \\
11 & 561672 & 1485 & 38720 & 46342 & 112364 & 87 & 281 & 862 & 2454
\end{tabular}

have a look at how the structure of $G$ evolves when perforating an $\alpha$-permutation.

Lemma 7.1. For every $k$-perforation $\pi$ of an $\alpha$-permutation $\sigma$ in $S_{n+k}$ :

$$
c(G(\pi))=c_{\text {odd }}(G(\pi))=k
$$

and $G(\pi)$ contains only noncrossing cycles, not containing each other, except for a large one containing all others.

Proof. Induction on $k$. The main cycle of $\Gamma(\sigma)$ is again assumed to be increasing, the decreasing case

TABLE 2

Number of Cases where (13) Overestimates $d(\pi)$ by $\Delta$

\begin{tabular}{r|r|r|r|r|r|r}
$n$ & $n !$ & $\Delta=0$ & $\Delta=1$ & $\Delta=2$ & $\Delta=3$ & $\Delta=4$ \\
\hline 1 & 1 & 1 & 0 & 0 & 0 & 0 \\
2 & 2 & 1 & 1 & 0 & 0 & 0 \\
3 & 6 & 2 & 4 & 0 & 0 & 0 \\
4 & 24 & 11 & 11 & 2 & 0 & 0 \\
5 & 120 & 48 & 51 & 21 & 0 & 0 \\
6 & 720 & 197 & 401 & 108 & 14 & 0 \\
7 & 5040 & 1318 & 2460 & 966 & 296 & 0 \\
8 & 40320 & 8775 & 13875 & 15150 & 2512 & 8 \\
9 & 362880 & 45415 & 132257 & 145394 & 34702 & 5112
\end{tabular}

corresponding to $\sigma^{-1}$ whose cycle graph has the same structure (see Hultman [15]). Recall that $n+k$ is odd, by definition of $\sigma$.

If $k=1$, let us remove some fixed element $\sigma_{i}=i$ ( $i$ is therefore even) by first applying transposition $\tau(i-1, i, i+1)$. This transposition acts on two interleaving cycles of the same parity in $G$ (Observation 4.1) and is therefore a 0-move (Lemma 2.1), transforming those cycles into a 1-cycle and an $(n+k)$-cycle, both odd. We now remove the adjacency, and get a permutation $\pi$ with $c(G(\pi))=c_{\text {odd }}(G(\pi))=1$.

For the induction, we again remove 1-cycles from $\Gamma$ in two steps by first applying all our transpositions, then removing $k$ adjacencies. Since the thesis is assumed to hold for $k-1$ perforations, we start with the corresponding $(k-1)$-perforation $\pi^{\prime}$ and put back the $k-1$ adjacencies that needed to be deleted, thus obtaining a permutation $\pi^{\prime \prime}$ with $c\left(G\left(\pi^{\prime \prime}\right)=c_{\text {odd }}\left(G\left(\pi^{\prime \prime}\right)\right)=2(k-1)\right.$. None of these cycles cross, and one of them contains all others. Let us now select some even $\pi_{i}^{\prime \prime}$ we wish to remove and apply the adequate transposition $\tau$ to make it adjacent to $\pi_{i}^{\prime \prime}+1$. The odd alternating cycle to which this element belongs will be cut into three cycles: an adjacency (1-cycle) "framed" by two cycles. 

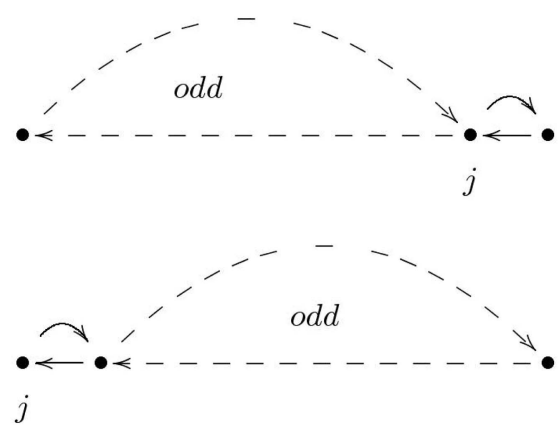

(a)

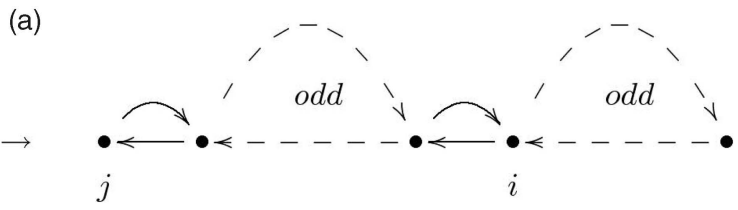

(b)

Fig. 6. Checking the parity of the new cycles in the proof of Lemma 7.1.

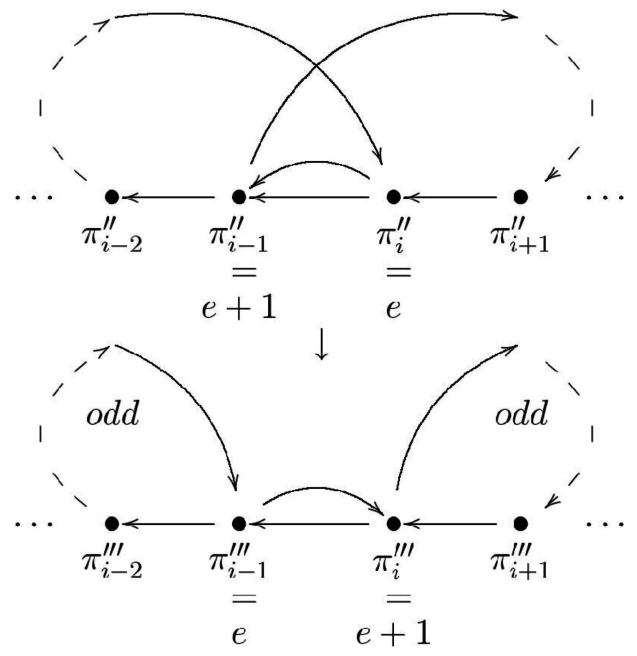

We need to prove that both framing cycles are odd, which comes down to showing that one of them is; indeed, the cycle we cut was odd, so the two cycles framing the new adjacency have the same parity. By induction, since at least one perforation has already been performed, there is an adjacency on the righthand side or on the left-hand side of the cut cycle. This adjacency is caused by an even element in an odd position and the number of black edges between an even position $i$ and an odd position $j$ is odd (Fig. 6 illustrates our claim). Therefore, the three new cycles are odd and we get the permutation $\pi^{\prime \prime \prime}=\pi^{\prime \prime} \circ \tau$ with $c\left(G\left(\pi^{\prime \prime \prime}\right)\right)=c_{\text {odd }}\left(G\left(\pi^{\prime \prime \prime}\right)\right)=2 k$. We now remove $k 1$-cycles from $G\left(\pi^{\prime \prime \prime}\right)$ and the proof follows.

This leads to a formula for computing the distance of such a permutation.

Corollary 7.1. For every $k$-perforation $\pi$ of an $\alpha$-permutation $\sigma$ in $S_{n+k}$ :

$$
d(\pi)=n-c_{\text {odd }}(\Gamma(\pi))-k+(|C| \bmod 2),
$$

where $|C|=\frac{n+k+1}{2}$ is the number of elements in its main cycle $C$.

Proof. Again, assume without loss of generality that $C$ is increasing; Lemma 7.1 and Theorem 2.1 yield $d(\pi) \geq|C|-k$. It is easily seen that removing a fixed point from $\Gamma(\sigma)$ replaces the edge of length 2 that overhangs it in $C$ with an edge of length 1 , so $C$ contains

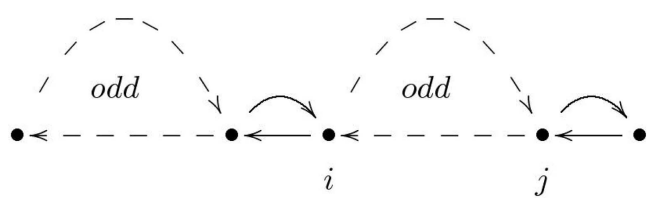

$k$ edges of length 1 and $\frac{n+k+1}{2}-k-1=\frac{n-1-k}{2}$ edges of length 2 (the last one has length $n$ ). Using $\frac{n-1-k}{2}$ transpositions of the form $\tau(i, i+1, i+2)$, where $i$ is the starting point of an edge of length 2, we transform $\pi$ into (2 $345 \cdots n+k-2 n+k-1 n+k 1)$ which is one transposition away from $\iota$. We therefore apply

$$
\frac{n-1-k}{2}+1=\frac{n+k+1}{2}-k=|C|-k
$$

transpositions in order to sort $\pi$, which completes the proof since

$$
\begin{aligned}
d(\pi) & =|C|-k \\
& =|C|-k-(|C| \bmod 2)+(|C| \bmod 2) \\
& =d(\sigma)-k+(|C| \bmod 2) \\
& =n+k-c_{\text {odd }}(\Gamma(\sigma))-k+(|C| \bmod 2) \\
& =n-c_{\text {odd }}(\Gamma(\sigma))+(|C| \bmod 2) \\
& =n-c_{\text {odd }}(\Gamma(\pi))-k+(|C| \bmod 2)
\end{aligned}
$$

The next logical move, as in our analysis of $\gamma$-permutations, would be to consider perforations of $\beta$-permutations. However, counterexamples have been found that prevent us from proving an equivalent of Lemma 7.1 in the case of those permutations; for instance, consider the $\beta$-permutation (72 13 $43658151091211141)$. Then, the cycle graph of the

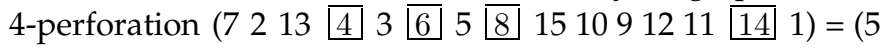
21034117698 1) has only two cycles, both odd.

We can nevertheless still study permutations whose $\Gamma$-graph contains noncrossing cycles only. Fortunately, the exact distance of some subcases in that family can be computed; if not, we are nonetheless still able to improve (11).

Before tackling this general problem in the next section, we conclude this one with the particular case where all noncrossing long cycles are perforations of $\alpha$-permutations, starting with the case shown in Fig. 7, where we do not allow containment of long cycles. In such a configuration,

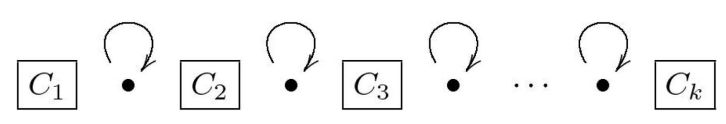

Fig. 7. A $\Gamma$-graph formed by subpermutations separated by 1-cycles. 

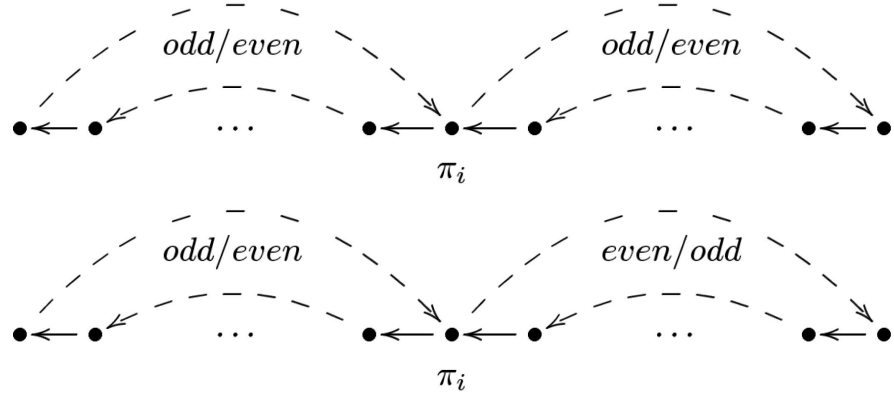

Fig. 8. Proof of Lemma 7.2.

the 1-cycles between every pair of long cycles are referred to as the separating 1-cycles or, more concisely, the separators.

Proposition 7.1. Let $\pi$ in $S_{n}$ be a permutation with $\Gamma(\pi)$ of the form shown in Fig. 7, where $C_{i}(1 \leq i \leq k)$ is a $k_{i}$-perforation of an $\alpha$-permutation; then,

$$
d(\pi)=n-c_{\text {odd }}(\Gamma(\pi))-K+\sum_{i=1}^{k}\left(\left|C_{i}\right| \bmod 2\right),
$$

where $K=\sum_{i=1}^{k} k_{i}$ and $\left|C_{i}\right|$ is the number of elements in the main cycle of each perforation.

Proof. Lemma 7.1 and Theorem 2.1 yield

$$
d(\pi) \geq \frac{n+1-\sum_{i=1}^{k} k_{i}}{2}=\frac{n+1-K}{2} .
$$

We have $n=k-1+\sum_{i=1}^{k} n_{i}$, where $n_{i}$ is the number of elements of each perforation and Lemma 4.2 and Corollary 7.1 yield

$$
\begin{aligned}
d(\pi) \leq \sum_{i=1}^{k} d\left(C_{i}\right) & =\sum_{i=1}^{k}\left|C_{i}\right|-k_{i} \\
& =\sum_{i=1}^{k} \frac{n_{i}+k_{i}+1-2 k_{i}}{2} \\
& =\frac{1}{2} \sum_{i=1}^{k}\left(n_{i}+1-k_{i}\right) \\
& =\frac{n+1-K}{2} .
\end{aligned}
$$

The expression given in the thesis is obtained by replacing $d\left(C_{i}\right)$ with the expression provided by Corollary 7.1.

We now show that removing any subset of the separators in the case we just examined does not affect the distance. For any transposition $\tau$ and any permutation $\pi$, let $\Delta c_{\text {odd }}(\tau, G(\pi))=c_{\text {odd }}(G(\pi \circ \tau))-c_{\text {odd }}(G(\pi))$. The following lemma will be useful:

Lemma 7.2. Let $\tau=\tau\left(i, i+1, \pi_{\pi_{i}+1}^{-1}\right)$ and let $C_{1}, C_{2}$ be two cycles in $G(\pi)$ which share vertex $\pi_{i}$, as shown below:

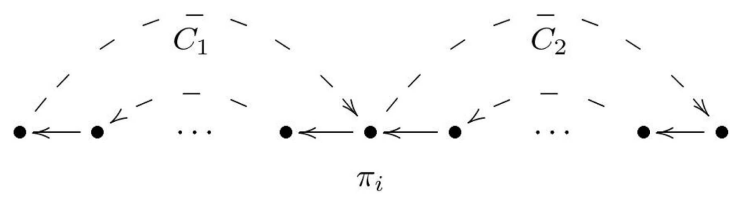

Then, $\Delta c_{\text {odd }}(\tau, G(\pi))=2$ if both $C_{1}$ and $C_{2}$ are even and 0 otherwise.

Proof. Fig. 8 shows the four cases.

Corollary 7.2. Let $\pi$ be a permutation that satisfies the conditions of Proposition 7.1; then, removing $j(1 \leq j \leq k-1)$ separators from $\Gamma(\pi)$ yields a permutation with the same distance.

Proof. By Lemma 7.1, each $C_{i}$ in $\Gamma(\pi)$ corresponds to a collection of alternating cycles in $G(\pi)$ wrapped in a large one and all of them are odd. Every pair of consecutive "wrapping cycles" in $G(\pi)$ shares a vertex, which is the 1-cycle separating the corresponding long cycles in $\Gamma(\pi)$. By Lemma 7.2, deleting that separating cycle does not change the bounds obtained in Proposition 7.1 and the proof follows.

We refer to subpermutations reducing to $\alpha$-permutations as $\alpha$-cycles. Similar arguments can be used to handle the case of cycles in $\Gamma$ that contain other ones, so we have the following result:

Theorem 7.1. For every $\pi$ in $S_{n}$ whose $\Gamma$-graph contains only 1 -cycles and $k$ noncrossing perforations of $\alpha$-cycles:

$$
d(\pi)=n-c_{\text {odd }}(\Gamma(\pi))-K+\sum_{i=1}^{k}\left(\left|C_{i}\right| \bmod 2\right),
$$

where $K$ is the number of edges of length 1 in $\Gamma(\pi)$.

Proof. The formula follows from Proposition 7.1 and previous observations. The correspondence with 1-edges in $\Gamma(\pi)$ was observed in the proof of Corollary 7.1, and this is the only case where deleting a 1-cycle creates an edge of length 1 .

It is less clear how exactly a perforation would be defined in the case of crossing cycles. Even less clear is the evolution of cycles in $G$ when deleting fixed points in this situation: It depends on how the cycles cross and on their monotonicity. We can, however, prove some further results on permutations whose $\Gamma$-graph has no crossing cycles, which we do in the next section.

\section{Noncrossing Cycles in $\Gamma$}

We consider permutations with a $\Gamma$-graph of the form shown in Fig. 7 and have a look at what happens in $G$ and $\Gamma$ when deleting separators. Depending on the parity of each 

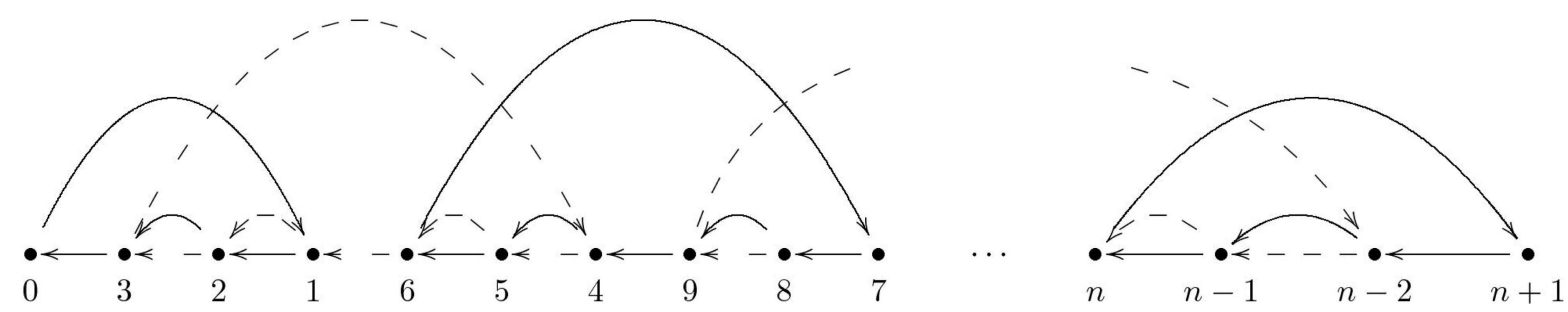

Fig. 9. The cycle graph of $\xi_{k}$.

long cycle, the deletion of separators can have various effects.

Proposition 8.1. Let $\pi$ in $S_{n}$ be a permutation with $\Gamma(\pi)$ of the form shown in Fig. 7, where $C_{i}(1 \leq i \leq k)$ is one of the following:

- $\quad$ an $\alpha$-permutation with an odd main cycle,

- a $\beta$-permutation with an odd main cycle, and

- a perforation of an $\alpha$-permutation.

Then, deleting $j$ separators $(1 \leq j \leq k-1)$ transforms $\pi$ into a permutation with the same distance.

Proof. By Propositions 4.1 and 4.5, we have

$$
d(\pi)=\frac{n+1-c_{\text {odd }}(G(\pi))}{2} .
$$

Each pair $\left(C_{i}, C_{i+1}\right)$ yields a pair of alternating cycles (Observation 4.1 and Lemma 7.1) that share the separator as described in Lemma 7.2. This lemma also implies that deleting the separator does not change the lower bound of Theorem 2.1, which is tight for $\pi$, because it will decrease both $n$ and the number of odd alternating cycles by 1 . So, $d(\pi)$ is a lower bound on the distance of the resulting permutation and, since $d(\pi)$ is also an upper bound on that distance (Lemma 4.2), the proof follows. $\square$

Although we are unable to compute the exact distance when all large cycles are even (and are not perforations of $\alpha$-permutations), we can still lower (11) in that case. In order to express this improved bound formally, we need to introduce the following graph:

Definition 8.1. Given a permutation $\pi$ with $\Gamma(\pi)$ of the form shown in Fig. 7, the contact graph $H(\pi)$ is the undirected graph whose vertices are the long cycles in $\Gamma(\pi)$ and whose edges are $\left\{C_{i}, C_{i+1}\right\}$ if $C_{i}$ and $C_{i+1}$ are even and not separated by a 1-cycle in $\Gamma(\pi)$.

This graph uniquely decomposes into $p$ connected components, which we denote $\mathcal{C}_{1}, \ldots, \mathcal{C}_{p}$. The following lemma will be useful:

Lemma 8.1. Let

$$
\xi_{k}=(\underbrace{321}_{1} \underbrace{654}_{2} \cdots \underbrace{n n-1 n-2}_{k}),
$$

then $d\left(\xi_{k}\right) \leq\left\lceil\frac{3 k}{2}\right\rceil=\left\lceil\frac{n}{2}\right\rceil$.
Proof. Since $\xi_{k}$ is a simple permutation (Fig. 9), the proof follows from Theorem 2.7.

One way to sort $\xi_{k}$ is to handle 2-cycles of $\Gamma\left(\xi_{k}\right)$ pairwise, i.e., partition $\xi_{k}$ into $\left\lfloor\frac{k}{2}\right\rfloor$ subpermutations of the form of $\xi_{2}$. Those can each be sorted optimally using three transpositions and, possibly, one last subpermutation of the form of $\xi_{1}$ will require two transpositions. Note that this permutation is the general form of an example given by Christie [5] that shows how his improved lower bound on the transposition distance fails (meaning that even though it gives a larger value than the lower bound of Theorem 2.1, it still underestimates the true distance). Branch-and-bound seems, however, to indicate that the upper bound of Lemma 8.1 is the actual distance of $\xi_{k}$.

Proposition 8.2. Let $\pi$ be a $\gamma$-permutation with $\Gamma(\pi)$ of the form shown in Fig. 7, where $C_{i}(1 \leq i \leq k)$ is either an $\alpha$-permutation or a $\beta$-permutation with an even main cycle, then deleting $j$ separators $(1 \leq j \leq k-1)$ transforms $\pi$ into a permutation $\sigma$ such that

$$
d(\sigma) \leq d(\pi)-2 k+\sum_{i=1}^{p}\left\lceil\frac{3\left|\mathcal{C}_{i}\right|}{2}\right\rceil,
$$

where $\mathcal{C}_{i}(1 \leq i \leq p)$ is a connected component of $H(\sigma)$.

Proof. Instead of removing separators directly, we first apply some transpositions on $\pi$. Each sub- $\alpha$-permutation can be sorted "incompletely" using the optimal sorting sequence of Proposition 4.2, without the last two transpositions. A similar process can be applied to sub- $\beta$-permutations, which first require a transformation as depicted in the proof of Proposition 4.4. By reduction, the resulting permutation has a $\Gamma$-graph of the form shown in Fig. 7, where each $C_{i}^{\prime}$ is now of the form of $\xi_{1}$. Let us now remove a subset of $j$ separators $(1 \leq j \leq k-1)$ from that permutation; this will diminish the number of components in its contact graph, thus creating subpermutations of the form of $\xi_{k}$. The following upper bound is obtained from Lemmas 4.2 and 8.1 : 


$$
\begin{aligned}
d(\sigma) & \leq \sum_{i=1}^{p} d\left(\mathcal{C}_{i}\right) \\
& \leq \sum_{i=1}^{p}\left(\sum_{C_{j} \in \mathcal{C}_{i}}\left(d\left(C_{j}\right)-2\right)+d\left(\xi_{\left|\mathcal{C}_{i}\right|}\right)\right) \\
& =\sum_{i=1}^{p} \sum_{C_{j} \in \mathcal{C}_{i}}\left(d\left(C_{j}\right)-2\right)+\sum_{i=1}^{p} d\left(\xi_{\left|\mathcal{C}_{i}\right|}\right) \\
& \leq d(\pi)-2 k+\sum_{i=1}^{p}\left\lceil\frac{3\left|\mathcal{C}_{i}\right|}{2}\right\rceil .
\end{aligned}
$$

An easy particular case of this proposition is when all separators are deleted; in that case, $d(\sigma) \leq d(\pi)-\left\lceil\frac{k}{2}\right\rceil$. There remains one case to deal with, which encompasses both previous propositions.

Proposition 8.3. Let $\pi$ be a $\gamma$-permutation with $\Gamma(\pi)$ of the form shown in Fig. 7, where $C_{i}(1 \leq i \leq k)$ is one of the following:

- an $\alpha$-permutation or a $\beta$-permutation with an even or an odd main cycle and

- a perforation of an $\alpha$-permutation.

Then deleting $j$ separators $(1 \leq j \leq k-1)$ transforms $\pi$ into a permutation $\sigma$ such that

$$
d(\sigma) \leq d(\pi)-2 k+\sum_{i=1}^{p}\left\lceil\frac{3\left|\mathcal{C}_{i}\right|}{2}\right\rceil,
$$

where $\mathcal{C}_{i}(1 \leq i \leq p)$ is a connected component of $H(\sigma)$.

Proof. As hinted by Lemma 7.2 and confirmed by previous results, the only case in which deleting a separator affects the distance of the resulting permutation is when that deletion occurs between two even cycles. This means that Proposition 8.2 naturally generalizes to the case where some cycles are allowed to be odd because deleting separators adjacent to at least one long odd cycle will not modify the distance of the resulting permutation. By the same arguments as those used in Proposition 8.2's proof, we obtain the same upper bound on the distance of the resulting permutation, and $\alpha$-permutations, $\beta$-permutations as well as perforations of the former kind can be handled individually in $\sigma$ as was already done in $\pi$.

We conclude with the case where we allow containment and perforation of $\alpha$-cycles.

Theorem 8.1. For all $\pi$ in $S_{n}$ with $\Gamma(\pi)$ containing only noncrossing $\alpha$-cycles that are odd or perforated (possibly both) and 1-cycles, we have

$$
d(\pi)=n-c_{\text {odd }}(\Gamma(\pi))-K+\sum_{i=1}^{k}\left(\left|C_{i}\right| \bmod 2\right),
$$

where $C_{i}(1 \leq i \leq k)$ are the long cycles in $\Gamma(\pi)$ and $K$ is the number of edges of length 1.

Proof. Suppose that every pair of consecutive long cycles in $\Gamma(\pi)$ is separated by a 1-cycle; since each long cycle is odd or a perforation of an $\alpha$-permutation, the corresponding alternating cycles in $G(\pi)$ are all odd (Proposition 4.1 and Lemma 7.1). Therefore, removing any subset of the separators cannot affect the distance (Lemma 7.2), so the strategy of Lemma 4.2 remains optimal and the proof follows from Theorem 7.1.

\section{Conclusions}

We have exhibited connections between two different graph representations of permutations, one of which is a well-known object in combinatorics and the other one is the traditional structure used in the problem of sorting permutations by transpositions. Those connections allowed us to derive a formula for computing the distance of a nontrivial class of permutations, which we called $\gamma$-permutations. Showing how $\gamma$-permutations could be used to generate all others, we were able to prove that our formula is an upper bound on the transposition distance of every permutation. A more involved analysis of the operation used to obtain other permutations from this class allowed us to describe three additional interesting families of permutations: more instances for which our bound is tight, instances for which our bound is not tight, but for which we found other formulas to compute their distance, and, finally, instances for which we can lower our upper bound without a guarantee that the obtained formula gives the exact distance.

It should be noted that (10) gives the distance of more permutations than the ones characterized in Theorem 4.1: Among the other permutations for which (10) still holds are 1-perforations of $\alpha$-permutations with an odd main cycle (Corollary 7.1), permutations obtained by concatenating such configurations, whether they are separated (Proposition 7.1) or not (Corollary 7.2), and permutations characterized in Proposition 8.1. Our results can also be used as upper bounds in some cases where cycles cross, for which it seems difficult to give an accurate formula or a more precise upper bound.

A few questions remain open. Although we now have a large quantity of permutations whose distance is computable in polynomial time, there are still some instances for which we have no clear answer yet. Among those are perforations of $\beta$-permutations, and permutations whose $\Gamma$-graph contains only crossing cycles and do not reduce to $\gamma$-permutations. Is it possible to compute their distance in polynomial time or to show it is NP-hard to do it? Can an improved upper bound be given as well?

An obviously related question is that of finding the diameter, i.e., the maximal value the transposition distance can reach. Using permutations whose distance we know, can we give an improved upper bound on the distance of permutations that do not belong to these families and, therefore, improve the upper bound of Theorem 2.9? 


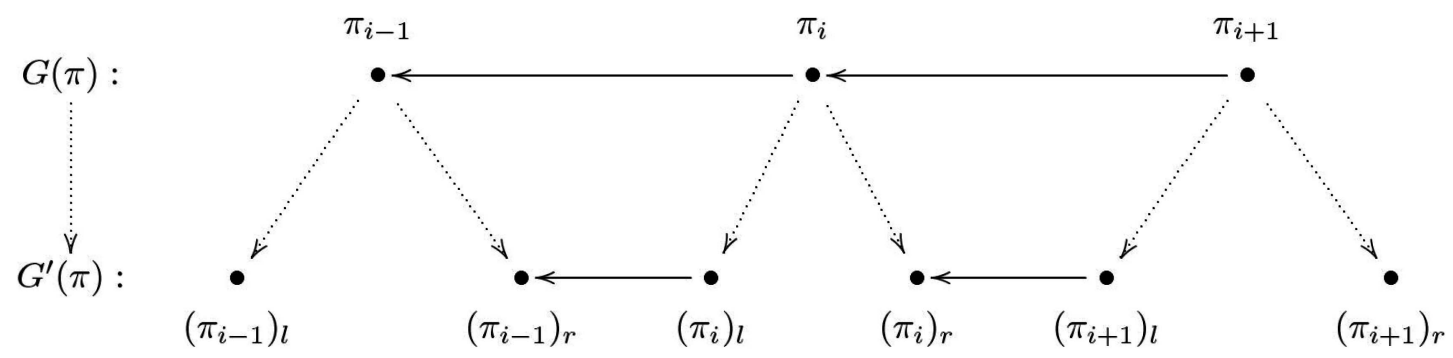

Fig. 10. Mapping of the black edges in the transformation of $G(\pi)$ into $B G\left(\pi^{\prime}\right)$; here, $G^{\prime}(\pi)$ is a graph that will be isomorphic to $B G\left(\pi^{\prime}\right)$ once the orientation of edges is removed.

\section{APPENDIX A}

\section{ON the Sequences of Proposition 4.2}

Consider the following transpositions:

$$
\left\{\begin{array}{l}
\tau_{1}=\tau(2,4, n+1) \\
\tau_{2}=\tau(1,3, n) \\
\tau_{3}=\tau(2,3, n+1) \\
\tau_{4}=\tau(1,2, n+1)
\end{array}\right.
$$

Proposition A.1. For every $\alpha$-permutation $\pi$ in $S_{n}$ whose main cycle $C$ is odd and increasing, the sequence

$$
\left(\tau_{2} \circ \tau_{1}\right)^{\frac{|C|-1}{2}}
$$

sorts $\pi$.

Proof. Induction on $|C|$. The base case is $\pi=\left(\begin{array}{l}325 \\ 2\end{array}\right.$ have $\pi \circ \tau_{1}=\left(\begin{array}{lllll}3 & 4 & 1 & 2 & 5\end{array}\right)$, and (3 $\left.4 \begin{array}{llll}4 & 1 & 2 & 5\end{array}\right) \circ \tau_{2}=\iota$.

For the induction, the permutation to sort is

$$
\pi=(325476 \cdots n-2 n-3 n n-11) .
$$

Applying $\tau_{1}$ to $\pi$ transforms it into

$$
(3476 \cdots n-2 n-3 n n-1125)
$$

to which we apply $\tau_{2}$, thus transforming it into

$$
(76 \cdots n-2 n-3 n n-112345) \text {. }
$$

Reducing the latter permutation merges the last five elements into a new element called 1 and subtracts 4 from every other element. It is then clear that, if $\sigma$ is the permutation for which our induction hypothesis is true, then $\pi \circ \tau_{1} \circ \tau_{2} \equiv_{r} \sigma$, and this completes the proof.

Proposition A.2. For every $\alpha$-permutation $\pi$ in $S_{n}$ whose main cycle $C$ is even and increasing, the sequence

$$
\tau_{4} \circ \tau_{3} \circ\left(\tau_{2} \circ \tau_{1}\right)^{\frac{|C|-2}{2}}
$$

sorts $\pi$.

Proof. Similar to that of Proposition A.1, with base case

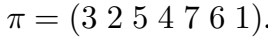

\section{APPENDIX B \\ On the Definition of Simple Permutations, 2-Permutations, and 3-Permutations}

A signed permutation is a permutation whose elements can be either positive or negative. Denote $S_{n}^{ \pm}$the group of permutations of $\{ \pm 1, \pm 2, \ldots, \pm n\}$. It is not mandatory for a signed permutation to have negative elements, so $S_{n} \subset S_{n}^{ \pm}$. The following graph was introduced by Bafna and Pevzner [18] in the context of sorting permutations by reversals.

Definition B.1. Given a signed permutation $\pi$ in $S_{n}^{ \pm}$, transform it into an unsigned permutation $\pi^{\prime}$ in $S_{2 n}$ by replacing $\pi_{i}$ with the sequence $\left(2 \pi_{i}-1,2 \pi_{i}\right)$ if $\pi_{i}>0$ or $\left(2\left|\pi_{i}\right|, 2\left|\pi_{i}\right|-1\right)$ if $\pi_{i}<0$, for $1 \leq i \leq n$. The breakpoint graph of $\pi^{\prime}$ is the undirected bicolored graph $B G\left(\pi^{\prime}\right)$ with ordered vertex set $\left(\pi_{0}^{\prime}=0, \pi_{1}^{\prime}, \pi_{2}^{\prime}, \ldots, \pi_{2 n}^{\prime}, \pi_{2 n+1}^{\prime}=2 n+1\right)$ and whose edge set consists of

- black edges $\left\{\pi_{2 i}^{\prime}, \pi_{2 i+1}^{\prime}\right\}$ for $0 \leq i \leq n$ and

- gray edges $\left\{\pi_{2 i}^{\prime}, \pi_{2 i}^{\prime}+1\right\}$ for $0 \leq i \leq n$.

We show that, for every signed permutation $\pi$ with no negative element, the cycle graph $G(\pi)$ is equivalent to the breakpoint graph $B G\left(\pi^{\prime}\right)$. By equivalent, we mean that every alternating cycle in $G(\pi)$ is an alternating cycle in $B G\left(\pi^{\prime}\right)$ and that the "topological" relations between the cycles are the same; for instance, if two cycles cross in either graph, then they also cross in the other one.

Theorem B.1. For all $\pi$ in $S_{n}: G(\pi) \equiv B G\left(\pi^{\prime}\right)$.

Proof. We show that either graph can be constructed by transforming the other one without affecting its features. Intuitively, transforming $G(\pi)$ into $B G\left(\pi^{\prime}\right)$ is done by spacing black edges in $G(\pi)$ and removing the orientation; conversely, transforming $B G\left(\pi^{\prime}\right)$ into $G(\pi)$ is done by orienting edges in $B G\left(\pi^{\prime}\right)$, then merging every consecutive pair of vertices that are not connected by a black edge.

1. Starting with $G(\pi)$ : Split each vertex $\pi_{i}(1 \leq i \leq n)$ into two unconnected vertices $\left(\pi_{i}\right)_{l},\left(\pi_{i}\right)_{r}$ (one to the left and one to the right), and rename $\pi_{0}$ (respectively, $\pi_{n+1}$ ) into $\left(\pi_{0}\right)_{r}$ (respectively, $\left.\left(\pi_{n+1}\right)_{l}\right)$. Black edge $\left(\pi_{i}, \pi_{i-1}\right)$ is mapped onto a new black edge $\left(\left(\pi_{i}\right)_{l},\left(\pi_{i-1}\right)_{r}\right)$, as shown in Fig. 10. Similarly, gray edge $\left(\pi_{i}, \pi_{i}+1\right)$ is mapped onto a new gray edge $\left(\left(\pi_{i}\right)_{r},\left(\pi_{i}+1\right)_{l}\right)$, as shown in Fig. 11. Finally, rename $\left(\pi_{i}\right)_{l}$ (respectively, 


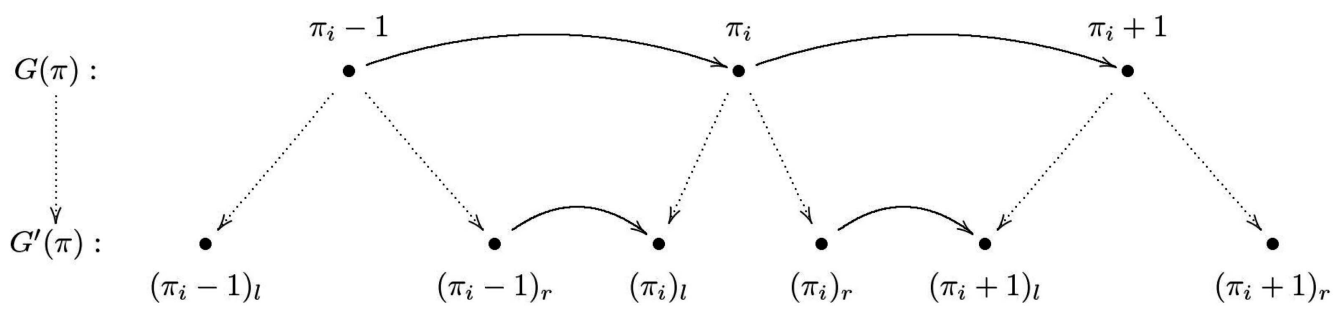

Fig. 11. Mapping of the gray edges in the transformation of $G(\pi)$ into $B G\left(\pi^{\prime}\right)$.

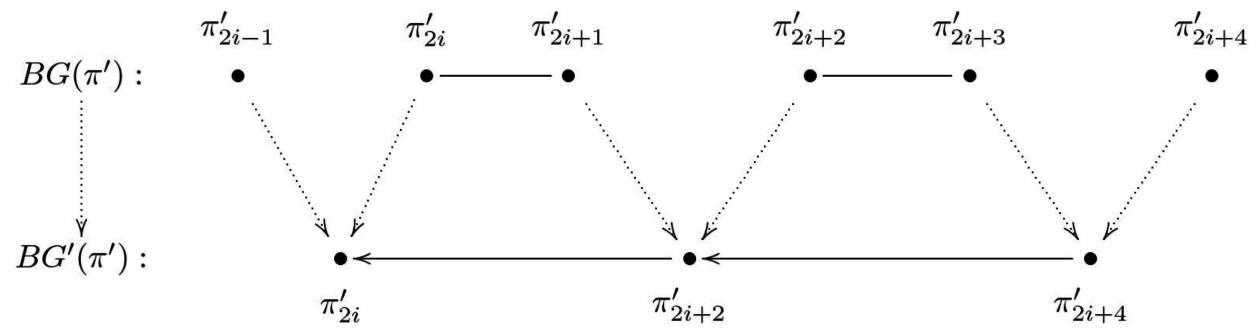

Fig. 12. Mapping of the black edges in the transformation of $B G\left(\pi^{\prime}\right)$ into $G(\pi)$; here, $B G^{\prime}\left(\pi^{\prime}\right)$ is a graph isomorphic to $G(\pi)$.

$\left(\pi_{i}\right)_{r}$ ) into $2 \pi_{i}-1$ (respectively, $2 \pi_{i}$ ) and remove the orientation of edges. This results in $B G\left(\pi^{\prime}\right)$ since:

a. each black edge $\left(\pi_{i}, \pi_{i-1}\right)$ is mapped onto a black edge $\left\{\left(\pi_{i}\right)_{l},\left(\pi_{i-1}\right)_{r}\right\}=\left\{2 \pi_{i}-1,2 \pi_{i-1}\right\}$ and

b. each gray edge $\left(\pi_{i}, \pi_{i}+1\right)$ is mapped onto a gray edge $\left\{\left(\pi_{i}\right)_{r},\left(\pi_{i}+1\right)_{l}\right\}=\left\{2 \pi_{i}, 2 \pi_{i}+1\right\}$.

2. Starting with $B G\left(\pi^{\prime}\right)$ : Since $\pi^{\prime}$ comes from some permutation $\pi$ with no negative element, for all $1 \leq i \leq n$, we have $\pi_{2 i}^{\prime}=2 \pi_{i}$ and $\pi_{2 i-1}^{\prime}=2 \pi_{i}-1$. This implies that alternating cycles in $B G\left(\pi^{\prime}\right)$ can be followed starting from the leftmost vertex of a black edge, then following a gray edge that will take us to the rightmost vertex of the next black edge. Therefore, adding an orientation to all edges that corresponds to this course will result in a collection of directed alternating cycles that can be followed using the direction of the arrows and this orientation is obtained by transforming gray edge $\left\{\pi_{2 i}^{\prime}, \pi_{2 i}^{\prime}+1\right\}$ into $\left(\pi_{2 i}^{\prime}, \pi_{2 i}^{\prime}+1\right)$ and black edge $\left\{\pi_{2 i}^{\prime}, \pi_{2 i+1}^{\prime}\right\}$ into $\left(\pi_{2 i+1}^{\prime}, \pi_{2 i}^{\prime}\right)$.

Next, for $1 \leq i \leq n$, merge vertices $\pi_{2 i-1}^{\prime}$ and $\pi_{2 i}^{\prime}$ into vertex $\pi_{2 i}^{\prime}$ and rename vertex $\pi_{2 n+1}^{\prime}$ into $\pi_{2 n+2}^{\prime}$; black edge $\left(\pi_{2 i+1}^{\prime}, \pi_{2 i}^{\prime}\right)$ is mapped onto a new black edge $\left(\pi_{2 i+2}^{\prime}, \pi_{2 i}^{\prime}\right)$, as shown in Fig. 12 .

Finally, replace $\pi_{2 i}^{\prime}$ with $\pi_{i}$, for $0 \leq i \leq n+1$. This results in $G(\pi)$ since:

a. each black edge $\left\{\pi_{2 i}^{\prime}, \pi_{2 i+1}^{\prime}\right\}$ is mapped onto a black edge $\left(\pi_{2(i+1)}^{\prime}, \pi_{2 i}^{\prime}\right)=\left(\pi_{i+1}, \pi_{i}\right)$ and

b. each gray edge $\left\{\pi_{2 i}^{\prime}, \pi_{2 i}^{\prime}+1\right\}$ is mapped onto a grey edge $\left(\pi_{i}, \pi_{i}+1\right)$.
As in the case of the cycle graph, the length of a cycle in a breakpoint graph is the number of black edges it contains.

Definition B.2 [17]. A permutation $\pi$ in $S_{n}^{ \pm}$is simple if $B G\left(\pi^{\prime}\right)$ does not contain a cycle of length greater than three.

Definition B.3 [17]. A permutation $\pi$ in $S_{n}^{ \pm}$is a 2-permutation (respectively, 3-permutation) if all cycles in $B G\left(\pi^{\prime}\right)$ are of length 2 (respectively, 3).

Corollary B.1. For every $\pi$ in $S_{n}$, Definition 2.16 (respectively, Definition 2.17) and Definition B.2 (respectively, Definition B.3) are equivalent.

Proof. Straightforward from Theorem B.1.

\section{ACKNOWLEDGMENTS}

The author is funded by the "Fonds pour la Formation à la Recherche dans l"Industrie et dans l'Agriculture" (F.R.I.A.).

\section{REFERENCES}

[1] J. Meidanis and J. Setubal, Introduction to Computational Molecular Biology. Brooks-Cole, 1997.

[2] P.A. Pevzner, Computational Molecular Biology. MIT Press, 2000

[3] V. Bafna and P.A. Pevzner, "Sorting Permutations by Transpositions," Proc. Sixth Ann. ACM-SIAM Symp. Discrete Algorithms, pp. 614-623, Jan. 1995.

[4] V. Bafna and P.A. Pevzner, "Sorting by Transpositions," SIAM J. Discrete Math., vol. 11, no. 2, pp. 224-240, May 1998.

[5] D.A. Christie, "Genome Rearrangement Problems," PhD dissertation, Univ. of Glasgow, Scotland, Aug. 1998.

[6] T. Hartman and R. Shamir, "A Simpler and Faster 1.5-Approximation Algorithm for Sorting by Transpositions," Information and Computation, vol. 204, no. 2, pp. 275-290, Feb. 2006.

[7] I. Elias and T. Hartman, "A 1.375-Approximation Algorithm for Sorting by Transpositions," Proc. Fifth Workshop Algorithms in Bioinformatics, R. Casadio and G. Myers, eds., pp. 204-214, Oct. 2005.

[8] S.A. Guyer, L.S. Heath, and J.P. Vergara, "Subsequence and Run Heuristics for Sorting by Transpositions," Fourth DIMACS Algorithm Implementation Challenge, Rutgers Univ., Aug. 1995. 
[9] J.P.C. Vergara, "Sorting by Bounded Permutations," PhD dissertation, Virginia Polytechnic Inst., Blacksburg, Apr. 1997.

[10] M.E.M.T. Walter, L.R.A.F. Curado, and A.G. Oliveira, “Working on the Problem of Sorting by Transpositions on Genome Rearrangements," Proc. 14th Ann. Symp. Combinatorial Pattern Matching, pp. 372-383, 2003.

[11] Z. Dias and J. Meidanis, "An Alternative Algebraic Formalism for Genome Rearrangements," Comparative Genomics: Empirical and Analytical Approaches to Gene Order Dynamics, Map Alignment and the Evolution of Gene Families, vol. 1, pp. 213-223, 2000.

[12] Z. Dias and J. Meidanis, "Genome Rearrangements Distance by Fusion, Fission, and Transposition Is Easy," Proc. Eighth Int'l Symp. String Processing and Information Retrieval, pp. 250-253, Nov. 2001.

[13] A. Labarre, "A New Tight Upper Bound on the Transposition Distance," Proc. Fifth Workshop Algorithms in Bioinformatics, R. Casadio and G. Myers, eds., pp. 216-227, Oct. 2005.

[14] H. Eriksson, K. Eriksson, J. Karlander, L. Svensson, and J. Wästlund, "Sorting a Bridge Hand," Discrete Math., vol. 241, nos. 1-3, pp. 289-300, 2001.

[15] A. Hultman, "Toric Permutations," master's thesis, Dept. of Math., KTH, Stockholm, Sweden, 1999.

[16] Z. Dias, J. Meidanis, and M.E.M.T. Walter, "A New Approach for Approximating the Transposition Distance," Proc. Seventh Int'l Symp. String Processing and Information Retrieval, pp. 199-208, Sept. 2000.

[17] S. Hannenhalli and P.A. Pevzner, "Transforming Cabbage into Turnip: Polynomial Algorithm for Sorting Signed Permutations by Reversals," J. ACM, vol. 46, no. 1, pp. 1-27, Jan. 1999.

[18] V. Bafna and P.A. Pevzner, "Genome Rearrangements and Sorting by Reversals," Proc. 34th Annual Symp. Foundations of Computer Science, pp. 148-157, 1993.

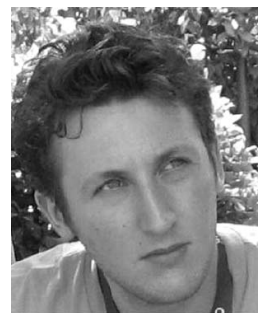

Anthony Labarre received the master's degree in computer science in 2004 and the DEA degree (Diplôme d'Études Approfondies) in sciences in 2005, both from the Université Libre de Bruxelles, Brussels, Belgium. He is currently working on a $\mathrm{PhD}$ thesis. His research interests include genome rearrangements, phylogenetic networks, and enumerative combinatorics.

$\triangleright$ For more information on this or any other computing topic, please visit our Digital Library at www.computer.org/publications/dlib. 